\title{
Identification of CO Adsorption Sites in Supported Pt Catalysts Using High-Energy-Resolution Fluorescence Detection X-ray Spectroscopy
}

\author{
Olga V. Safonova, ${ }^{\dagger}$ Moniek Tromp, ${ }^{*, \ddagger}$ Jeroen A. van Bokhoven, ${ }^{*} \S$ Frank M. F. de Groot, \\ John Evans, $\stackrel{\ddagger}{ }$, and Pieter Glatzel ${ }^{\dagger}$ \\ European Synchrotron Radiation Facility, 6 rue Jules Horowitz, 38043 Grenoble, France, School of Chemistry, \\ University of Southampton, Southampton, SO17 1BJ, United Kingdom, Institute for Chemical and \\ Bioengineering, ETH Zurich, 8093 Zurich, Switzerland, Department of Inorganic Chemistry and Catalysis, \\ Sorbonnelaan 16, 3584 CA, Utrecht, The Netherlands, and Diamond Light Source, Chilton, \\ Didcot, OX11 0QX, United Kingdom
}

Received: June 2, 2006; In Final Form: July 6, 2006

\begin{abstract}
High-energy-resolution fluorescence detection (HERFD) X-ray spectroscopy is presented as a new tool for the identification of the bonding sites of reactants in supported metal catalysts. The type of adsorption site of $\mathrm{CO}$ on an alumina-supported platinum catalyst and the orbitals involved in the bonding are identified. Because X-ray absorption spectroscopy (XAS) is element-specific and can be used under high pressures and temperatures, in situ HERFD XAS can be applied to a swathe of catalytic systems, including alloys.
\end{abstract}

Metal nanoparticles are used in many catalytic processes such as selective and total oxidation and hydrogenation reactions. The reactivity of nanoparticles depends greatly on their size, morphology, and the nature of the support. Understanding the correlation between the structure of the active sites and the performance of the catalytic system is extremely important for the design of better catalysts to increase conversion and selectivity to a desired reaction product. However, no characterization technique is capable of providing all information on the structures of catalytic sites under reaction conditions on real catalyst particles. Here, we present high-energy-resolution fluorescence detection (HERFD) X-ray spectroscopy as a new tool for the identification of the bonding sites of reactants in supported metal catalysts. The types of adsorption sites of $\mathrm{CO}$ on platinum on alumina catalyst and the orbitals involved in the bonding are identified.

The geometry of the $\mathrm{CO}$ adsorption site on perfect and stepped single crystal surfaces has been intensively studied by vibration spectroscopy, ${ }^{1}$ low-energy electron diffraction (LEED), ${ }^{2}$ and scanning tunneling microscopy. ${ }^{3}$ Three main $\mathrm{CO}$ adsorption configurations on Pt surfaces were identified: atop (one-fold), bridged (two-fold), and face bridging (three-fold). The overall conclusion is that $\mathrm{CO}$ prefers coordination to a single $\mathrm{Pt}$ atom at the lowest coverage and the atop-bridge combination at high coverage. ${ }^{4}$ The picture is similar for oxide-supported platinum nanoparticles, however, the support composition, its acidity, and pore curvature influence the $\mathrm{CO}$ adsorption geometry. ${ }^{5-9}$

The sensitivity of the near-edge structure of X-ray absorption spectra (XANES) of metal nanoparticles to the presence of

* To whom correspondence should be addressed. E-mail: m.tromp@ soton.ac.uk; J. A.vanBokhoven@chem.ethz.ch.

$\dagger$ European Synchrotron Radiation Facility.

$\div$ University of Southampton.

$\S$ Institute for Chemical and Bioengineering.

"Department of Inorganic Chemistry and Catalysis.

$\perp$ Diamond Light Source. adsorbed molecules and the correlation between the shapes of Pt L edges and the geometry of the hydrogen and oxygen adsorption sites in supported Pt nanoparticles have been reported. ${ }^{10}$ According to the optical dipole selection rule, $\mathrm{L}_{2,3}$ X-ray absorption spectroscopy probes predominantly the $\mathrm{d}$ projected empty density of states (DOS). The intensity of the first feature in the $\mathrm{L}_{3}$ edge spectrum (the whiteline) probes the number of holes in the d-band. Changes in the XANES after molecular adsorption originate from orbital rehybridization, charge transfer, metal-absorbate scattering, and differences in metal-metal scattering. Intrinsic broadening of the experimental spectra occurs because of the finite lifetime of the core hole, rendering the experimental XAS spectra much broader than the actual DOS. For example, the lifetime broadening of the Pt $2 \mathrm{p}_{3 / 2}$ $\left(\mathrm{L}_{3}\right)$ core level measured by XPS is equal to $5.3 \mathrm{eV}$. ${ }^{11 a}$ To detect subtle variations in the XANES region, detection of XAS spectra with higher resolution is required. The first attempts to overcome this fundamental limitation were undertaken by Hämäläinen, ${ }^{11 b}$ who observed the spectral sharpening of a Dy XANES spectrum by monitoring only the Dy $L \alpha_{1}$ fluorescence with an energy resolution better than the core hole lifetime broadening. De Groot and co-workers measured the Pt L edges by monitoring $\mathrm{L} \beta$ fluorescence lines with $2 \mathrm{eV}$ resolution ${ }^{12}$ and showed that HERFD spectra have a much better resolved fine structure compared to the XANES spectra measured in transmission mode. We have recently shown this to be similar for $\mathrm{Au} \mathrm{L}_{3}$ HERFD XANES (monitoring $L \alpha_{1}$ ), providing insights in the activation of oxygen and its reaction with $\mathrm{CO}$ over an $\mathrm{Au} / \mathrm{Al}_{2} \mathrm{O}_{3}$ catalyst. $^{13}$

Here, we present the first application of HERFD X-ray absorption spectroscopy as a probe of the bonding sites of adsorbed molecules on the surface of supported nanoparticles. The details on the synthesis of the supported Pt nanoparticles, the in situ reactor, the experimental conditions, and the theoretical calculations are given in the Supporting Information. 


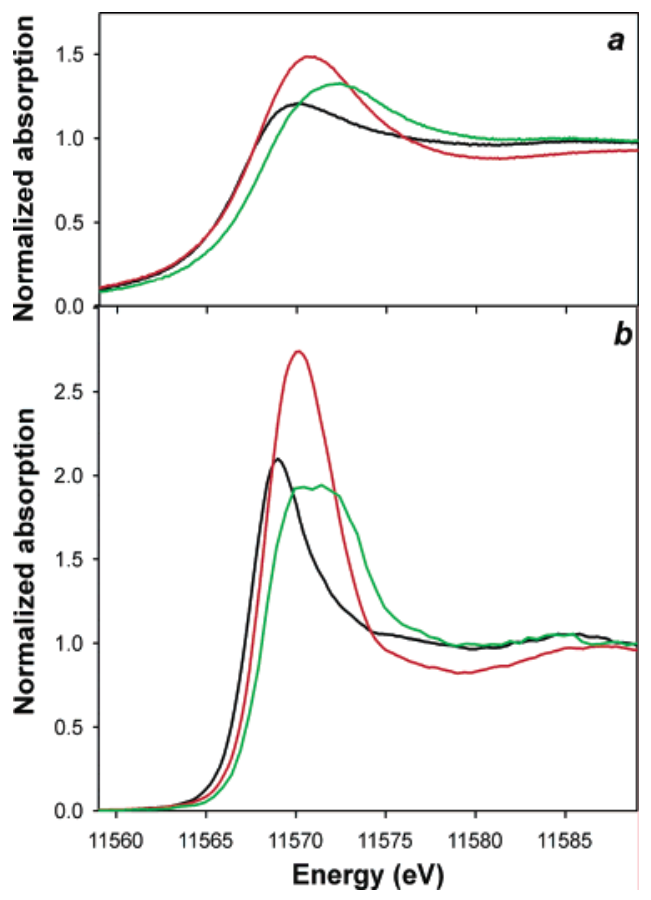

Figure 1. $\mathrm{Pt} \mathrm{L}_{3}$ edge of $5 \mathrm{wt} \% \mathrm{Pt} / \mathrm{Al}_{2} \mathrm{O}_{3}$ after reduction in the presence of different atmospheres: $\mathrm{He}$ (black), $\mathrm{He}\left(\mathrm{O}_{2}\right)$ (red), and $1 \% \mathrm{CO} / \mathrm{He}$ (green) measured using total fluorescence detection (a) and HERFD XAS spectroscopy (b).

The experiments were performed at the high brilliance XAFSXES beamline ID26, at the European Synchrotron Radiation Facility (ESRF) in Grenoble, France. The incident energy was selected by means of a pair of $\mathrm{Si}(220)$ crystals with an energy bandwidth of $0.7 \mathrm{eV}$. The HERFD was performed with a horizontal plane Rowland circle spectrometer tuned to the $\mathrm{Pt}$ $\mathrm{L} \alpha_{1}(9442 \mathrm{eV})$ fluorescence line. An energy bandwidth of 1.0 $\mathrm{eV}$ in the emission detection was achieved using the (660) Bragg reflection of one spherically bent Ge wafer. An avalanche photodiode (APD) was used as a detector. A Canberra silicon photodiode was mounted to measure the total fluorescence simultaneously.

Figure 1 compares the near-edge structure of the $\mathrm{Pt}_{2}$ edge of $5 \mathrm{wt} \% \mathrm{Pt} / \mathrm{Al}_{2} \mathrm{O}_{3}$ measured under different conditions using total fluorescence detection and HERFD. The sample was reduced in $\mathrm{H}_{2}$ at $473 \mathrm{~K}$. The $\mathrm{H}_{2}$ was subsequently removed in $\mathrm{He}$ at $473 \mathrm{~K}$. The XANES spectra, see Figure 1, were recorded in situ under steady-state conditions in, respectively, $\mathrm{He}, \mathrm{He}$ with traces of $\mathrm{O}_{2}\left(\mathrm{He}\left(\mathrm{O}_{2}\right)\right)$, and in $1 \% \mathrm{CO} / \mathrm{He}$ at $298 \mathrm{~K}$. A change in atmosphere leads to variations in the shape of the whiteline for both detection techniques. However, by the use of HERFD spectroscopy, these variations are more pronounced and better resolved. The spectrum of $\mathrm{Pt} / \mathrm{Al}_{2} \mathrm{O}_{3}$ after reduction shows the typical whiteline of reduced platinum particles. The intensity in the HERFD spectra, Figure 1b, is higher and structure becomes visible at $11576 \mathrm{eV}$. In both the total fluorescence detection and HERFD (parts a and b of Figure 1, respectively), the spectrum under $\mathrm{He}\left(\mathrm{O}_{2}\right)$ shows increased whiteline intensity and a loss of intensity at $10-20 \mathrm{eV}$ above the absorption edge, which is indicative of partial particle oxidation. Under a $1 \% \mathrm{CO} / \mathrm{He}$ atmosphere, the spectrum shows an energy shift and enhanced intensity in the whiteline that is broadened at the high-energy side. The HERFD data show the enhanced whiteline and reveal a double feature.

To identify the bonding sites of $\mathrm{CO}$, we performed calculations of the $\mathrm{Pt}_{3}$-edge spectra using the FEFF8. 2 code. ${ }^{14}$ Four different clusters were considered as models for the adsorption

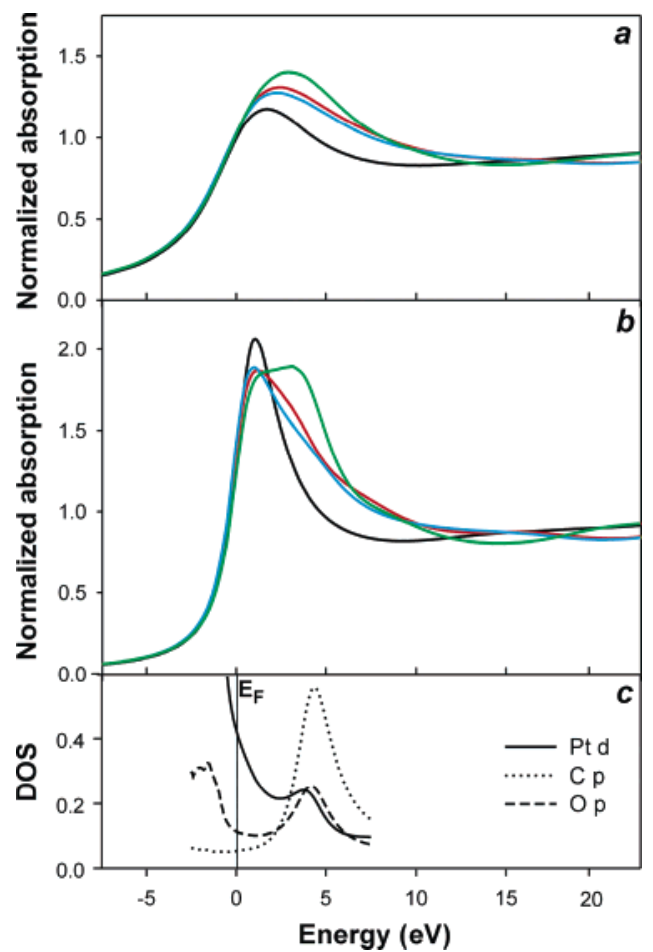

Figure 2. Theoretical (FEFF8.2) $\mathrm{Pt} \mathrm{L}_{3}$ edge of the $\mathrm{Pt}_{6}$ cluster (black) and the $\mathrm{Pt}_{6}-\mathrm{CO}$ clusters for $\mathrm{CO}$ atop (green), bridged (red), and face bridging (blue) with broadening (a) natural and (b) reduced by -1.6 $\mathrm{eV}$; (c) the d-DOS for Pt and p-DOS for $\mathrm{C}$ and $\mathrm{O}$ calculated for a $\mathrm{Pt}_{6}-\mathrm{CO}$ atop cluster.

sites: $\mathrm{Pt}_{6},{ }^{10 \mathrm{~b}} \mathrm{Pt}_{6}-\mathrm{CO}$ atop, $\mathrm{Pt}_{6}-\mathrm{CO}$ bridged, and $\mathrm{Pt}_{6}-\mathrm{CO}$ face bridging. Diagrams of the clusters are given in the Supporting Information. The lifetime broadenings of $\mathrm{L}_{3}$ and $\mathrm{M}_{5}$ levels tabulated in the FEFF8.2 code $^{14}$ are equal to 5.2 and $2.4 \mathrm{eV}$, respectively. According to the equation given in ref 12 , the broadening due to the core hole lifetimes is equal to $2.2 \mathrm{eV}$ in the HERFD spectra. The HERFD broadening (Lorentzian) is convoluted with the experimental broadening (Gaussian) and the width of Pt whiteline in the HERFD experiment should thus be close to $2.9 \mathrm{eV}$, which is similar to the value observed in the experiment. The line broadening in the FEFF calculations is given by the core hole lifetimes as well as the imaginary part of the self-energy. ${ }^{14}$ The line broadening can be reduced by using the EXCHANGE card in the FEFF input. The best agreement with experiment was achieved by using a value of vio $=-1.6 \mathrm{eV}$.

Parts a and $\mathrm{b}$ of Figure 2 show the theoretical spectra of the bare and $\mathrm{CO}$-containing platinum clusters without and with spectral sharpening, respectively. It was pointed out by Carra et al. ${ }^{15}$ that strong electron-electron interactions (multiplet effects) can render a HERFD spectrum different from an absorption spectrum. In particular, additional spectral features might appear or disappear that are not due to an absorption process. ${ }^{16}$ This effect is strong for localized orbitals such as valence band $3 \mathrm{~d}$ or $4 \mathrm{f}$ levels, and even in those cases, these effects are only visible if the overall resolution is below 1.0 eV. ${ }^{17}$ We did not observe any multiplet effects in the $2 p$ to $5 \mathrm{~d}$ transition (white line) and thus conclude that a HERFD spectrum gives a very good approximation of an absorption scan. Furthermore, the FEFF calculations accurately reproduce all HERFD spectral features.

The shape of the XANES in the spectrum of the reduced $\mathrm{Pt}_{6}$ cluster is similar to the experimental one with both detection techniques (Figures 1 and 2). The high-energy-resolution FEFF 
simulation (Figure 2b) of atop adsorbed CO on Pt shows the double feature in the whiteline, which is absent in the simulations of the other configurations. These calculations indicate that in $1 \% \mathrm{CO} / \mathrm{He}$ atmosphere, the predominant geometry of the $\mathrm{CO}$ adsorption site on Pt nanoparticles is atop rather than bridged or face bridging. This distinction is much more pronounced in the HERFD spectrum. The DOS calculated for the $\mathrm{Pt}_{6}-\mathrm{CO}$ atop cluster with FEFF are given in Figure $2 \mathrm{c}$. They show that d-orbitals on the metal are overlapping with the $2 \pi^{*}$ orbitals of the $\mathrm{C}$ and $\mathrm{O}$ atoms, forming an antibonding state above the Fermi level. This is reflected in the second feature of the whiteline in the HERFD spectrum of $\mathrm{Pt} / \mathrm{Al}_{2} \mathrm{O}_{3}$ in $1 \% \mathrm{CO} /$ $\mathrm{He}$ (Figure 1b). The transition has a substantial metal-to-ligand charge-transfer character. For $\mathrm{Pt}_{6}-\mathrm{CO}$ bridged and hollow sites, this component is weaker because the spatial distribution of the orbitals is less favorable for mixing.

In IR spectroscopy, probing the adsorbing molecule, variations in the stretching vibration of $\mathrm{CO}$ are related to the adsorption sites. ${ }^{6}$ In the HERFD XAS experiment, we can directly probe the structures of the adsorption sites and the DOS that are involved in the bonding. Therefore, HERFD and the vibrational spectroscopies provide complementary information and can be applied simultaneously. Because XAS is elementspecific and can be used under high pressures and temperatures, in situ HERFD can be applied to a swathe of catalytic systems, including alloys.

Acknowledgment. The authors acknowledge ESRF for provision of facilities at ID26 and ID24 (mass spectrometer) and financial support (EPSRC to M.T. and the Swiss National Science Foundation to J.A.vB.). They also acknowledge Dr. M. Sikora for help in the preparation of the HERFD experiment.

Supporting Information Available: Details on the synthesis of alumina-supported Pt nanoparticles, the in situ reactor, experimental conditions and theoretical calculations, and XAFS data under dynamic conditions. This material is available free of charge via the Internet at http://pubs.acs.org.

\section{References and Notes}

(1) (a) Eischens, R. P.; Plishkin, W. A. Adv. Catal. 1958, 10, 1. (b) Eischens, R. P.; Francis, S. A.; Plishkin, W. A. J. Phys. Chem. 1956, 60, 194. (c) Hollins, P. Surf. Sci. Rep. 1992, 16, 51. (d) Schweizer, E.; Persson, B. N. J.; Tushaus, M.; Hoge, D.; Bradshaw, A. M. Surf. Sci. 1989, 213, 49. (e) Hayden, B. E.; Kretschmar, K.; Bradshaw, A. M.; Greenler, R. G. Surf. Sci. 1985, 149, 394

(2) (a) Ogletree, D. F.; van Hove, M. A.; Somorjai, G. A. Surf. Sci. 1986, 173, 351. (b) Blackman, G. S.; et al. Phys. Rev. Lett. 1988, 61, 2352.

(3) (a) Bocquet, M. L.; Sautet, Ph. Surf. Sci. 1996, 360, 128. (b) Pedersen, M. O.; Bocquet, M. L.; Sautet, Ph.; Laegsgaard, E.; Stensgaard, I.; Besenbacher, F. Chem. Phys. Lett. 1999, 299, 403.

(4) Feibelman, P. J.; Hammer, B.; Nørskov, J. K.; Wagner, F.; Scheffler, M.; Stumpf, R.; Watwe, R.; Dumesic, J. J. Phys. Chem. B. 2001, 105, 4018.

(5) Mojet, B. L.; Miller, J. T.; Koningsberger, D. C. J. Phys. Chem. B 1999, 103, 2724.

(6) (a) Barth, R.; Pitchai, R.; Anderson, R. L.; Verykios, X. E. J. Catal. 1989, 116, 61. (b) Barth, R.; Ramachandran, A. J. Catal. 1990, 125, 467.

(7) Gandao, Z.; Coq, B.; de Menorval, L. C.; Tichit, D. Appl. Catal. A 1996, 147,395 .

(8) Fiddy, S. G.; Newton, M. A.; Campbell, T.; Dent, A. J.; Harvey, I.; Salvini, G.; Turin, S.; Evans, J. Phys. Chem. Chem. Phys. 2002, 4, 827. (9) Visser, T.; Nijhuis, T. A.; van der Eerden, A. M. J.; Jenken, K.; Ji, Y.; Bras, W.; Nikitenko, S.; Ikeda, Y.; Lepage, M.; Weckhuysen, B. M. J. Phys. Chem. B 2005, 109, 3822

(10) (a) Oudenhuijzen, M. K.; van Bokhoven, J. A.; Miller, J. T.; Ramaker, D. E.; Koningsberger, D. C. J. Am. Chem. Soc. 2005, 127, 1530. (b) Ramaker, D. E.; Teliska, M.; Zhang, Y.; Stakheev, A. Yu.; Koningsberger, D. C. Phys. Chem. Chem. Phys. 2003, 5, 4492. (c) Ankudinov, A. L.; Rehr, J. J.; Low, J. J.; Bare, S. R. Top. Catal. 2002, 18, 3.

(11) (a) Fuggle, J. C.; Inglesfield, J. E. Unoccupied Electronic States; Springer-Verlag: Berlin, 1992. (b) Hämäläinen, K.; Siddons, D. P.; Hastings, J. B.; Berman, L. E. Phys. Rev. Lett. 1991, 67, 2850.

(12) de Groot, F. M. F.; Krish, K. H.; Vogel, J. Phys. Rev. B. 2002, 66 , 195112

(13) van Bokhoven, J. A.; Louis, C.; Miller, J. T.; Tromp, M.; Safonova, O. V.; Glatzel, P. Angew. Chem., Int. Ed. 2006, 45 (28), 4651; Angew. Chem. 2006, 118, 4767.

(14) (a) Ankudinov, A. L.; Ravel, B.; Rehr, J. J.; Conradson, S. D. Phys. Rev. B 1998, 58, 998. (b) Ankudinov, A. L.; Bouldin, C.; Rehr, J. J.; Sims, J.; Hung, H. Phys. Rev. B 2002, 65, 104107.

(15) Carra, P.; Fabrizio, M.; Thole, B. T. Phys. Rev. Lett. 1995, 74 , 3700 .

(16) Glatzel, P.; Bergmann, U. Coord. Chem. Rev. 2005, 249, 65.

(17) de Groot, F. M. F.; Glatzel, P.; Bergmann, U.; van Aken, P. A.; Barrea, R. A.; Klemme, S.; Havecker, M.; Knop-Gericke, A.; Heijboer, W. M.; Weckhuysen, B. M. J. Phys. Chem. B 2005, 109, 20751. 